\title{
Phenol Adsorption onto Modified Industrial Solid Waste Adsorbents in the Presence of Cationic and Anionic Surfactant
}

\author{
TAN Gang ${ }^{1, a}$, XUE Yongjie ${ }^{2, b}$ and WAN Li, \\ ${ }^{1}$ School of Materials Science and Engineering, Wuhan University of Technology, China \\ ${ }^{2}$ State Key Laboratory of Silicate Materials for Architectures, Wuhan University of Technology, China \\ ${ }^{3}$ Cccc Wuhan Harbor Engineering Design and Research Co. Ltd. \\ atg666happy@126.com, bxyjskl@whut.edu.cn, ${ }^{\mathrm{c}}$ from702853@163.com
}

Keywords: Adsorption; Phenol; Industrial solid waste; Surfactants; Mechanism

\begin{abstract}
The objective of this study is to remove phenol from aqueous solution by using modified industrial solid waste (ISW) in the presence of cationic and anionic surfactant with batch adsorption. Tests were carried out as functions of contact time, initial phenol concentration, $\mathrm{pH}$ and temperature. The experiments demonstrated that maximum phenol adsorption removal was obtained in a wide $\mathrm{pH}$ range of 3-13 and it took $3 \mathrm{~h}$ to attain adsorption equilibrium. With an increase of temperature, phenol adsorption amount decreased. The mechanism of phenol adsorption onto ISW adsorbents in the presence of cationic and anionic surfactants was discussed. There are two main mechanisms. Mechanochemistry effect improved adsorption capacity of ISW adsorbents by having particles ground finer and activated; and then in the presence of surfactants, zero point charge of adsorbents surface was major factor to influence combination of adsorbents with surfactants and adsorption capacity of organic-ISW adsorbents
\end{abstract}

\section{Introduction}

Phenols, which are considered as priority pollutants since they are harmful to organisms at low concentrations and have been classified as hazardous pollutants, are popularly used in the preparation of antiseptics, dyes, antirust products, synthetic resins, biocides, photographic chemicals, ink, varnishes, etc. [1]. Phenols can cause severe odour and taste problems and pose risks to populations. Various treatment technologies such as adsorption [2, 3], photo-degradation [4], coagulation flocculation, chemical oxidation [5, 6], biological process [7], etc. are available for the removal of phenol from the wastewater. Basic Oxygen Furnace slag (BOFs) is a final waste material in the basic oxygen furnace steel making process. Coal fly ash (FA) is one of solid wastes largely produced from power generation. There are large annual amount of these industrial solid waste (ISW) and most of researches were conducted about engineering application aspect, hence, few of them focus on environmental remediation materials, such adsorbents used in removal of hazardous and toxic materials, for example, adsorption removal of phenol from aqueous solution in the presence of surfactant. Based on many previous research works, it is a novel way to removal phenol by using ISW as low cost adsorbents, as well as solid waste resource application field. For this purpose, the characteristics of surfactants and phenol adsorption onto modified ISW adsorbents' surface were investigated. And results reflected whole adsorption effects such as functions of contact time, initial phenol concentration, $\mathrm{pH}$ and temperature. The mechanism of phenol adsorption was discussed as well.

\section{Materials and Methods}

Materials. Original BOFs and FA used in this study generated from the local steel-making factory with two years setting time and power plant respectively. Physical properties and chemical compositions are shown in Table 1. All chemicals and reagents used were of analytical grade. Stock solutions of phenol were prepared from analytical grade chemicals in de-ionized water as adsorbate. 
The cationic and anionic surfactants, Hexadecyltrimethylammonium (HDTMA) bromide and sodium dodecylsulfate (SDS) were used as received. Solution of $0.1 \mathrm{M} \mathrm{NaOH}$ and $0.1 \mathrm{M} \mathrm{HNO}_{3}$ were used for $\mathrm{pH}$ adjustment. All working solutions were prepared by diluting the stock solutions with de-ionized water.

Table 1 Chemical compositions and physical properties of adsorbents and surfactants

\begin{tabular}{|c|c|c|c|c|c|}
\hline \multicolumn{3}{|c|}{ Chemical composition } & \multirow{2}{*}{\multicolumn{3}{|c|}{ Physical properties }} \\
\hline \multirow{2}{*}{ Oxides } & \multicolumn{2}{|c|}{ Content / \% } & & & \\
\hline & BOFs & FA & Index & BOFs & FA \\
\hline $\mathrm{SiO}_{2}$ & 13.71 & 40.20 & Bulk density $/ \mathrm{g} / \mathrm{cm}^{3}$ & $3.11 \sim 3.29$ & $2.28 \sim 2.65$ \\
\hline $\mathrm{CaO}$ & 45.41 & 12.50 & Moisture / \% & 1.18 & 0.85 \\
\hline $\mathrm{MgO}$ & 6.25 & 2.61 & Blain surface area $/ \mathrm{m}^{2} / \mathrm{kg}$ & $415 \sim 754$ & $320 \sim 530$ \\
\hline $\mathrm{Al}_{2} \mathrm{O}_{3}$ & 3.80 & 21.05 & $\mathrm{D}_{50} / \mathrm{mm}$ & $4.59 \sim 7.24$ & $6.92 \sim 9.27$ \\
\hline Total Fe ${ }^{\mathrm{a}}$ & 21.85 & 11.70 & $\begin{array}{l}\text { Percentage of meso-poros } \\
\text { (pore size } 2-50 \mathrm{~nm}) / \%\end{array}$ & $48.5 \sim 65.2$ & $45.2 \sim 72.0$ \\
\hline Surfactant & & Formula & Molecular weight & $\mathrm{g} / \mathrm{mol}$ & $\mathrm{C}^{\mathrm{c}} / \mathrm{mol} / \mathrm{L}$ \\
\hline HDTMA & & $\mathrm{C}_{19} \mathrm{H}_{42} \mathrm{~N}^{+}$ & 364.46 & & $9.0 \times 10^{-4}$ \\
\hline SDS & & $\mathrm{C}_{12} \mathrm{H}_{25} \mathrm{SO}^{-}$ & 272.38 & & $8.0 \times 10^{-3}$ \\
\hline
\end{tabular}

Adsorbents Preparation. There were two steps to describe adsorbents preparation process. First, the sieve size of BOFs and FA was controlled under $0.6 \mathrm{~mm}$ as original materials. Sample ST1 and ST2 meant that adsorbents were ground for 1 and 6 hours by vertical planetary ball mill, while the ratio of materials versus ball was 1:10 by mass; sample FT1 and FT2 meant that adsorbents were ground for 1 and 6 hours by same mill and condition. Sample SB and FB meant blank experiment used dry BOFs and FA original materials as adsorbent respectively. At the second step, surfactants, including HDTMA and SDS were directly added into phenol aqueous solution in which phenol initial concentration was known. The amounts of addition of surfactants needed to keep their concentration for the 1 and 1.5 CMC (critical micelle concentration). This procedure was named after surfactant-modified adsorbent procedure 1 (SMAP1). On the other hand, the adsorbents, ST1, ST2, FT1 and FT2 were chosen and pretreated by HDTMA and SDS surfactants before adsorption of phenol for the purpose of adsorption enhancement. Surfactant-modified ISW adsorbents were prepared by adding amounts of surfactant equivalent to $0.01 \mathrm{M}$ of concentration. Dried surfactant-modified adsorbents were pulverized with a mortar and pestle [8,9]. The HDTMA and SDS modified ISW adsorbents were marked with HS1, HS2, SS1, SS2, HF1, HF2, SF1 and SF2, respectively for the surfactant-modified BOFs and surfactant-modified FA, then stored in airtight containers until it were used in adsorption experiments. This procedure was remarked as SMAP2.

Adsorption study. The equilibrium isotherms were conducted by contacting a constant mass of adsorbents with difference of initial and equilibrium concentration. For determination of equilibrium contact time at $293.15 \mathrm{~K}$, accurately weighting $1.00 \mathrm{~g}$ adsorbent and adding into $250 \mathrm{~mL}$ Erlenmeyer flask with $100 \mathrm{~mL}, 100 \mathrm{mg} / \mathrm{L}$ concentration of phenol solution for shaking $24 \mathrm{~h}$ and storing. For studying isotherms, experiments were stared at $293.15 \mathrm{~K}$ with $100 \mathrm{~mL}$ phenol solution which concentration varied from 50 to $1500 \mathrm{mg} / \mathrm{L}$ and $10 \mathrm{~g} / \mathrm{L}$ adsorbents dosage. Keep the dosage of adsorbents in solution for constant changing within $5,10,20$, and $40 \mathrm{~g} / \mathrm{L}$ at $293.15 \mathrm{~K}$. Adsorption experiments were started with $100 \mathrm{mg} / \mathrm{L}$ of initial phenol concentration. With a similar procedure, the effect of $\mathrm{pH}$ on phenol adsorption was evaluated at $293.15 \mathrm{~K}$ in a series of experiments that used the same initial phenol concentration $(100 \mathrm{mg} / \mathrm{L})$ and adsorbent dosage was kept $10 \mathrm{~g} / \mathrm{L}$ while varying $\mathrm{pH}$ value from 3 to 11 by adjusting solution $\mathrm{pH}$ with $0.1 \mathrm{M} \mathrm{NaOH}$ and $\mathrm{HNO}_{3}$. The effect of temperature on phenol adsorption was evaluated and tested as described above while maintaining temperature at $30,40,50$, and $60^{\circ} \mathrm{C}(303.15 \mathrm{~K}, 313.15 \mathrm{~K}, 323.15 \mathrm{~K}$, and $333.15 \mathrm{~K}$ in Kelvin temperature). All flasks for experiments should be shaken constantly for $3 \mathrm{~h}$ (except for contact time determination) and stored under standard room condition for another $3 \mathrm{~h}$. Phenol concentration form all of experiments of the supernatant solution after centrifuged was determined by spectrophotometer 
at maximum absorption wavelength of phenol at $291 \mathrm{~nm}$. The equilibrium concentration (equilibrium adsorption capacity) of phenol was calculated according to the following equations:

$$
q_{e}=\left(C_{0}-C_{e}\right) V_{0} / m
$$

Where, at equilibrium for all equations, $q_{e}(\mathrm{mg} / \mathrm{g})$ means adsorption capacity of phenol; $C_{0}$ $(\mathrm{mg} / \mathrm{L})$ and $C_{e}(\mathrm{mg} / \mathrm{L})$ mean initial and equilibrium concentration of phenol solution; $V_{0}(\mathrm{~mL})$ and $m$ (g) mean initial solution volume and mass of adsorbents; $t$ (min) means contact time. And all experiments were performed in triplicate and average results were kept.

\section{Results and Discussion}

Effect of Contact Time. Fig. 1 shows that there are two regions in it, region 1 and region 2, before and after $180 \mathrm{~min}$ of contact time respectively. There was a plateau for nearly every curve when adsorption started for $3 \mathrm{~h}$. It was also concluded that adsorption capacity of FT2 was the highest among all of adsorbents and the poorest for blank experiment which used original BOFs as adsorbent. Since the phenol adsorption capacity of original FA was higher than ST2, it was thought that FA was a kind of more available adsorbent than BOFs for phenol adsorption without surfactant modified. But generally, adsorption effect by ISW was not distinct and obvious for the maximum phenol adsorption capacity was merely $3.2 \mathrm{mg} / \mathrm{g}$. Fig. 2 (a) and (b) show that surfactants, HDTMA and SDS, indirectly modified ISW adsorbents as described in SMAP1. Surfactants and phenol existed simultaneously in solution before adsorbents were added. It can be observed that utilization of surfactants leaded to sharply increase and improve phenol adsorption onto some ISW adsorbents. Results of adsorption capacity of adsorbents from SMAP2 are shown in Fig. 3. It can be concluded that phenol adsorption capacity increased sharply at beginning of experiments. During first 15 min, adsorption capacity could reach to $5.7 \sim 6.8 \mathrm{mg} / \mathrm{g}$ for all of adsorbents. Curves showed smooth and still at $180 \mathrm{~min}$ there was a plateau for all curves. By surfactant directly modified, adsorption capacity of all of adsorbents increased after adsorption experiments, the maximum removal rate of phenol reached to $90 \%$, and the minimum was $67 \%$.
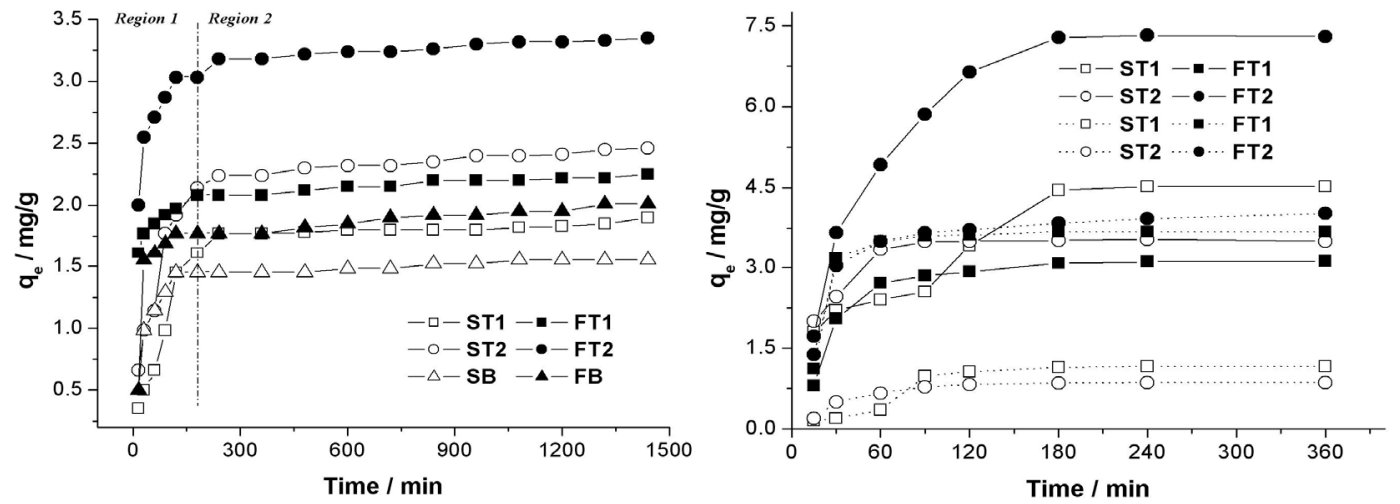

Fig. 1. Adsorption capacity versus contact time without surfactants.

Fig. 2. (a) Adsorption capacity versus contact time in the presence of HDTMA at SMAP1 ("_-" “........" meant concentrations of surfactant were 1.0 and $1.5 \mathrm{CMC}$; “口० •" meant ST1, ST2, FT1,

FT2 ISW adsorbents)
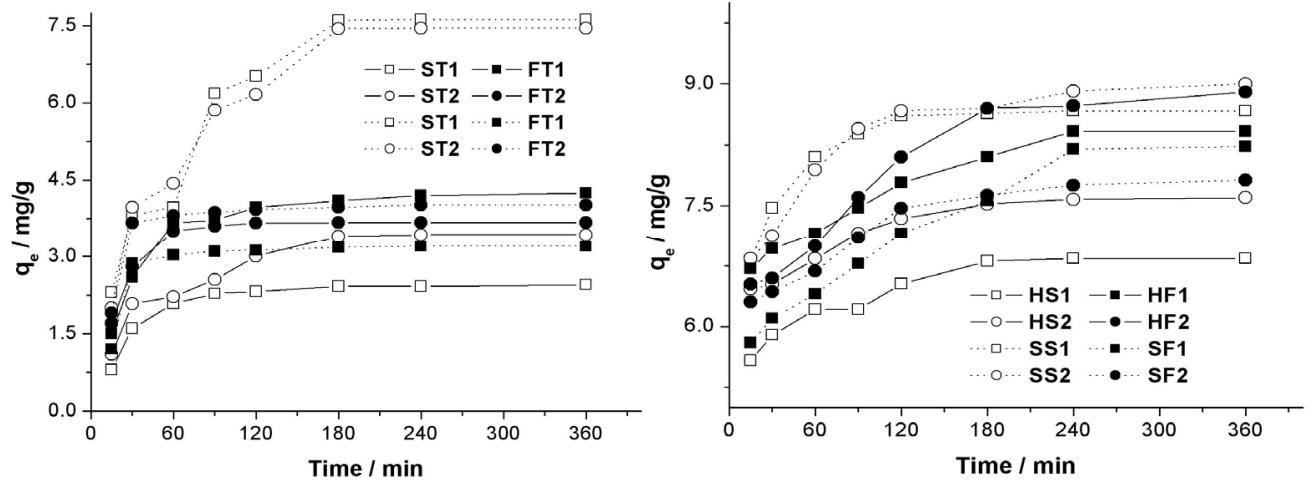
Fig. 2. (b) Adsorption capacity versus contact time in the presence of SDS at SMAP1 (“__ " “....... meant 1.0 and 1.5 concentrations of surfactant CMC; “口०п•" meant ST1, ST2, FT1, FT2 ISW adsorbents).

Fig. 3. Adsorption capacity versus contact time at SMAP2 (“_ "“....... " meant directly modified by 0.01M concentrations of HDTMA, SDS; “ø० •” meant HS1, HS2, SS1, SS2 ISW adsorbents).

Effect of Initial Concentration. Six kinds of ISW adsorbents modified by different methods were chosen to evaluate the effect of phenol initial concentration. It was found from Fig. 4 that two regions were divided to describe effect of different initial phenol concentrations onto adsorption capacity. In region 1, phenol adsorption capacity increased rapidly while equilibrium concentration of phenol increased slowly. When adsorption happened in region 2, capacity did not increase with an increase of initial concentration of phenol. Both in two regions, the amount of phenol adsorption capacity of adsorbents increased with increasing initial concentration. The initial concentration of phenol provided a force to overcome the resistances to the mass transfer of phenol between the aqueous and the solid phases of adsorbents. Also, the increase of initial phenol concentration enhanced the interaction between phenol and the ISW. It could be concluded that SS1 was the most efficient adsorbents while ST1 was not due to at same initial concentration, phenol adsorption capacity of the former was the highest while that of the later was the lowest.

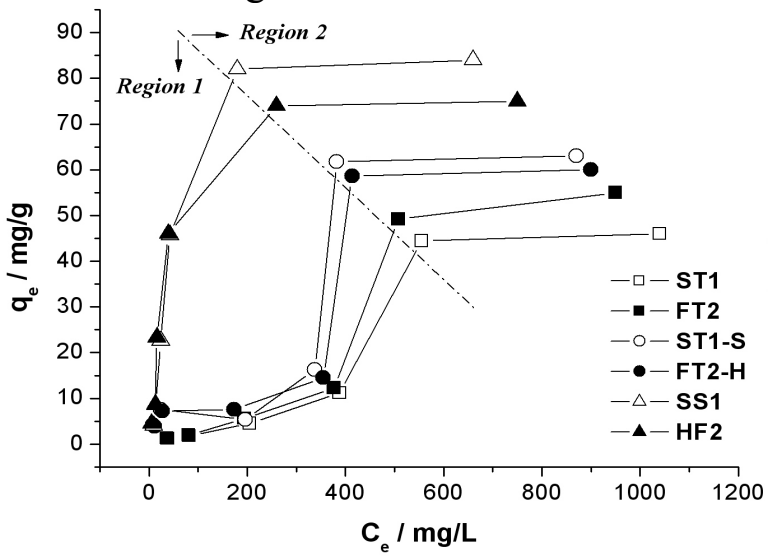

Fig. 4. Adsorption capacity versus equilibrium concentration of phenol

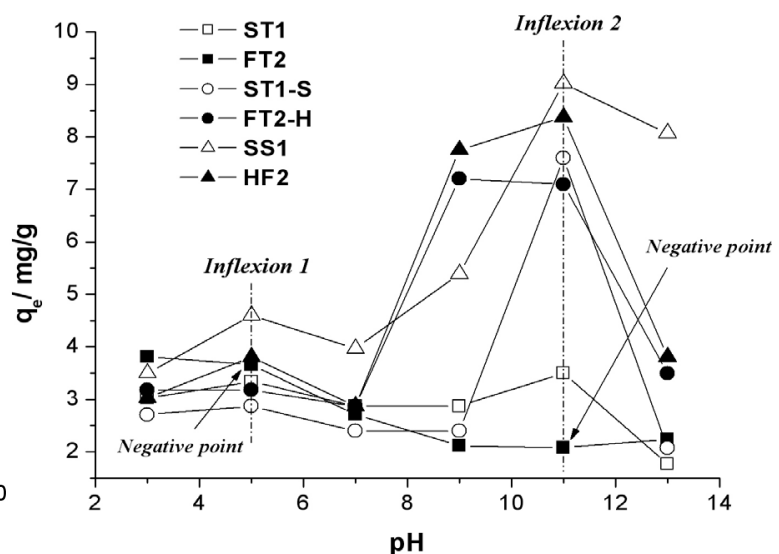

Fig. 5. Effect of $\mathrm{pH}$ on adsorption capacity

Effect of pH. The influence of $\mathrm{pH}$ of solution on the phenol adsorption is shown in Fig. 5. It was found that by regressing test results, there were two inflexions which showed the maximum adsorption amount in its region at $\mathrm{pH}=5$ and 11 respectively except for two negative points. Such curves could only provide general characteristics of effect of $\mathrm{pH}$ on phenol adsorption due to accuracy of experiment. But still it was concluded that there was a maximum amount of phenol adsorption at high $\mathrm{pH}$, and this $\mathrm{pH}$ of solution was close to 11; on the other hand, in acid environment, $\mathrm{pH}$ was close to 5 that seemed easier for adsorption removal of phenol from solution than that at other $\mathrm{pH}$ values below 7. From Fig. 5, it was observed that the adsorption of phenol by SS1 was slightly higher than any other adsorbents while adsorbents without surfactants modified showed lower phenol adsorption amount.

Effect of temperature. The effect of temperature on the extent of adsorption of phenol as a function of adsorption capacity is shown in Fig. 6. It was found that with an increase in temperature the phenol adsorption capacity of all adsorbents decreased except for three negative points in the curves. For ST1 and FT2, adsorbents modified by mechanochemistry, maximum adsorption capacity appeared at temperature $30^{\circ} \mathrm{C}$, while other four adsorbents appeared at $20^{\circ} \mathrm{C}$ that was normal experiment temperature. Since the adsorption is an exothermic process, it would be expected that an increase in temperature of the solid-liquid system would result in decrease adsorption capacity. Almost all of the experiment results fixed this rule but two points showed slight difference from other data. Besides, it seemed that SS1 and HF2, which showed higher efficiency of phenol adsorption removal, were influenced dramatically after temperature $30^{\circ} \mathrm{C}$. At any temperature after $30^{\circ} \mathrm{C}$, other four adsorbents showed slightly influenced. 


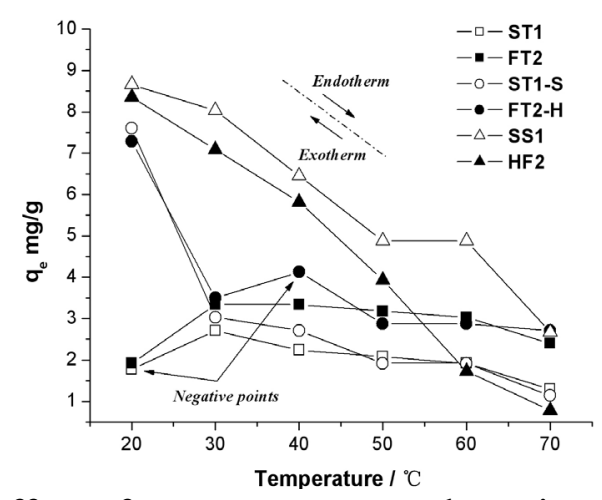

Fig. 6. Effect of Temperature on adsorption capacity.

Adsorption Mechanism. In this investigation, experiment results fitted for such a conclusion that un-modified ISW materials were not adsorbents which were available and efficient for phenol adsorption. Mechanochemistry effect had particle of ISW ground fine, higher surface area and porosity leaded to higher amount uptake of phenol. Phenol adsorption capacity of ground FA was higher than that of BOFs, and this was mainly due to agglomeration phenomena existed during materials being ground. Agglomeration phenomena did slight effect on particle of FA for adsorption removal phenol from Fig. 1. The surface and structure of ISW materials changed and was activated by mechanochemistry. Silicate tetrahedron $\left[\mathrm{SiO}_{4}\right]$ commonly exists in the structure of ISW materials, BOFs and FA, which contains silicate. It was found that the silicon oxide bond of silicate tetrahedron could be broken by mechanochemistry force to form monomer of silicate. Hence such effect leaded to activate surface of materials. Since $\mathrm{Si}-\mathrm{O}$ bond was broken and at every broken point $\mathrm{H}^{+}$and $\mathrm{OH}^{-}$ were adsorbed, surface was coated by $\equiv \mathrm{Si}-\mathrm{OH}$. Phenol $\left(\mathrm{C}_{6} \mathrm{H}_{6} \mathrm{O}\right)$ is weak acid in solution and ionizes $\mathrm{H}^{+}, \mathrm{C}_{6} \mathrm{H}_{5} \mathrm{O}^{-}$and can be absorbed by $\equiv \mathrm{Si}-\mathrm{OH}$.

After being modified by surfactants, it was easy to understand that surfactant had positive effect on organic materials adsorption onto inorganic materials, for example, phenol adsorption onto ISW. But as a result, different surfactant did different effect on adsorption. HDTMA had positive effect on phenol adsorption capacity of FA adsorbent, while SDS had positive effect on BOFs adsorbent from test results. This was thought to be explained by zero point charge of ISW materials. Due to ISW materials were mixture of all kinds of oxides. Test results of this study demonstrated that $\mathrm{pH}_{\mathrm{ZPC}}$ of BOFs and FA was 12.3 and 8.2. It was reported that at $\mathrm{pH}$ value of solution above the $\mathrm{pH}_{\mathrm{ZPC}}$, the solid surface of the adsorbents particle is negatively charged. Therefore, adsorption amount of cationic and anionic surfactants onto adsorbents were different due to its charge. Hence, it can be concluded that at $\mathrm{pH}$ lower than 12.3 for BOFs-phenol solution system, surface of BOFs showed positive charge; at $\mathrm{pH}$ higher than 8.2 for FA-phenol solution system, surface of FA was negatively charged. as a consequence, negatively charged SDS was easy to be absorbed onto BOFs surface due to electrostatic attraction while positively HDTMA was absorbed by FA due to same reason. With increasing amount of adsorbed surfactant onto adsorbents, a conglomeration of the alkyl chains on the surfaces and interlayer regions resulted in formation of an organic phase coated solid surfaces that controlled the adsorption of phenol adsorption from aqueous solution. Notice that results from Fig. 3 were higher than those from Fig. 2, which meant adsorbents modified from SMAP2 were more efficient than those from SMAP1. Fig. 7 indicated that difference between SMAP1 and SMAP2. In (a) for SMAP1, relationship between phenol and surfactant was competitive, even if there was electrostatic attraction between surfactant with adsorbents solid surface, phenol still had opportunity to be absorbed by adsorbents due to such attraction force was weak. This leaded to modification of adsorbents not complete and surfactant lost chance to bond with adsorbents, as a consequence, little amount of phenol was adsorbed due to its hydrophilic character to ISW materials. In (b) for SMAP2, it could be observed that every point of surface had been taken by surfactant due to pretreatment process. This would cause organic phase of adsorbents surface increased and as a result adsorption amount of phenol increased. 


\section{Summary}

The present study shows that the modified industrial solid wastes including fly ash and basic oxygen furnace slag are effective adsorbents for the removal of phenol from aqueous solutions. Adsorption equilibrium could be achieved in $3 \mathrm{~h}$. 32\% of higher removal rate was achieved for the adsorbents modified by mechanochemistry alone; while more than $76 \%$ was achieved in the presence of the surfactants. As a result, HDTMA had positive effect on phenol adsorption capacity of FA adsorbent, while SDS had positive effect on BOFs adsorbent. Experiment results showed that the direct modification by surfactant was more effective than the indirect. ISW could be used as adsorbents for phenol adsorption in a wide $\mathrm{pH}$ range of 3-13. It was concluded that there was a maximum amount of phenol adsorption at $\mathrm{pH}$ of 11 ; on the other hand, in acid environment, $\mathrm{pH}$ was close to 5 that seemed easier for adsorption removal of phenol from solution than that at other $\mathrm{pH}$ values below 7. Since the adsorption is an exothermic process, it was found that with an increase in temperature the phenol adsorption capacity of all adsorbents decreased. It seemed that adsorption by adsorbents from SMAP2 were influenced dramatically after temperature $30 \mathrm{C}$. This was due to various active sites or heterogeneous mixture of several minerals or oxides on ISW after ground had different affinities to phenol molecule. Higher surface area and porosity of ISW adsorbents led to increase of phenol adsorption capasity, while adsorption higher amount of cationic and anionic surfactants onto adsorbents due to different charge triggered off same results.

\section{References}

[1] F.A. Banat, B. Al-Bashir, S. Al-Ashes, O. Hayajnesh: Environ. Pollut. Vol. 107 (2000), p. 391

[2] S. Rengaraj, S.H. Moon, R. Sivabalan, B. Arabindoo, V. Murugesan: Waste Manage. Vol. 22 (2002), p. 543

[3] S. Haydar, M.A. Ferro-Garcia, J. Rivera-Utrilla: Carbon Vol. 41 (2003), p. 387

[4] C. Wu, X. Liu, D. Wei, I. Fan, L. Wang: Water Res. Vol. 35 (2001), p. 3927

[5] C.B. Maugans, A. Akgerman: Water Res. Vol. 31(1997), p. 3116

[6] H. Shon, S. Vigneswaran, H.H. Ngo, J. H. Kim: Water Res. Vol. 39 (2005), p. 2549

[7] C.S.A. Sa, R.A.R. Boaventura: Biochem. Eng. J. Vol. 9 (2001), p. 211

[8] I. Barros Valentim, I. Joekes: Coll. Surf. A: Physicochem. Eng. Aspects Vol. 29 (2006), p. 106

[9] Petr Praus, Martina Turicova, Sona Studentova, Michal Pitz: J. Colloid Interf. Sci. Vol. 304 (2006), p. 29 